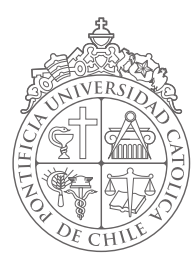

Todos los interesados en publicar en la Revista deben enviar su manuscrito a la dirección electrónica aporia@uc.cl o a su correo postal / Aporía invites any interested parties to contribute to the Journal by sending an article to the Director's electronic address: aporia@uc.cl or to his office by post:

Aporía. Revista Internacional de Investigaciones Filosóficas.

Facultad de Filosofía,

Pontificia Universidad Católica de Chile.

Campus San Joaquín. Av. Vicuña Mackenna 4860. Macul.

CP. 7820436.

Santiago de Chile (CHILE)

Información sobre Política editorial, Presentación de los manuscritos, Normas de estilo,

Derechos de autor y otros detalles, se hallan en el sitio: / Information concerning

Editorial Policy, Guidelines for contributors, Submission of articles, Copyright and other details are available at the web site: http://filosofia.uc.cl

Para suscripciones y canjes, dirigirse a: aporia@uc.cl o al correo postal de la revista. Subscription and Exchange orders may be made to: aporia@uc.cl or to its postal address.

Valor del ejemplar: $\$ 10.000$ pesos chilenos.

Price of the number: U\$20. - American dollars.

(C) Pontificia Universidad Católica de Chile. Todos los derechos reservados /

(C) Pontifical Catholic University of Chile. All rights reserved.

Diseño y Diagramación / Design and Layout:

Bookdesign - www.bookdesign.cl

Edición / Publisher:

Andros Impresores

Mayo 2020

Santiago de Chile

La Revista está indizada en:

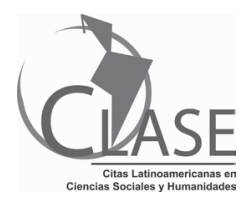




\section{CONTENIDOS / CONTENTS}

\section{ARTÍCULOS/ARTICLES}

ERIC POMMIER:

"EL FENÓMENO DE LA VIDA: BAJO EL PRISMA DE HANS JONAS

Y DE MAURICE MERLEAU-PONTY" 4-18

JOSÉ MIGUEL HERRERA Y ROSARIO ATHIÉ:

"FUNDAMENTOS DE LA SUBSIDIARIEDAD EN SIMONE WEIL:

AUTORIDAD Y AUTONOMÍA"

ESTEBAN SEPÚlVEDA CARO:

"EL DESEO Y SU EXPERIENCIA: UN EXAMEN DE LAS LECTURAS

DE ROBERT PIPPIN, FREDERICK NEUHOUSER Y AXEL HONNETH" $36-50$

CAMILO SILVA:

"LEIBNIZ Y EL LABERINTO DE LA NADA:

LA ONTOLOGÍA EN MANOS DE UN ESCOLÁSTICO FURTIVO" $51-74$

\section{RESEÑAS/BOOK REVIEWS}

EGUZKI URTEAGA:

"TOURAINE, A. (2018): DÉFENSE DE LA MODERNITÉ.

384 PP. PARIS: SEUIL" $76-90$

\section{NOTAS/REMARKS}

JOSÉ TOMÁS ALVARADO MARAMBIO:

"UNIVERSALES Y FUNDACIÓN ASCENDENTE. ACERCA DE T. SCOTT DIXON (2018), "UPWARD GROUNDING", PHILOSOPHY AND PHENOMENOLOGICAL RESEARCH 97, 1, PP. 48-78” 
ARTÍCULOS/ARTICLES 


\title{
EL FENÓMENO DE LA VIDA: BAJO EL PRISMA DE HANS JONAS Y DE MAURICE MERLEAU-PONTY
}

\author{
Prof. Dr. Eric Pommier \\ Pontificia Universidad Católica de Chile
}

\begin{abstract}
Resumen: Después de mostrar cómo la cuestión del ser conduce a la cuestión de la vida y a la exigencia de una fenomenología de la vida, presentamos un estudio comparativo entre la noción merleau-pontyana de comportamiento y la noción jonasiana de metabolismo. Esta equiparación abre a una crítica jonasiana del dispositivo merleau-pontyano y a una mejor determinación de los requisitos para proponer una descripción del fenómeno vital.
\end{abstract}

Descriptores: Comportamiento $\cdot$ Fenomenología $\cdot$ Metabolismo $\cdot$ Muerte $\cdot$ Vida.

\begin{abstract}
After showing how the question of the being leads to the question of life and the necessity of a phenomenology of life, we present a comparative study between the Merleaupontian notion of behavior and that of the Jonasian metabolism. Such a comparison opens a space to criticize the Merleau-pontian dispositive from a Jonasian point of view and makes it possible to improve the determination of conditions to propose a description of the phenomenon of life.
\end{abstract}

Keywords: Behavior $\cdot$ Death $\cdot$ Life $\cdot$ Metabolism $\cdot$ Phenomenology

Enviado: 09/01/2020. Aceptado: 14/04/2020

\section{Al Profesor Luis Flores Hernández, ${ }^{1}$}

La tarea que se asigna Hans Jonas consiste en retomar el problema del ser tal como la tradición nos lo legó. Eso significa, en una proximidad con el voto heideggeriano de destrucción, que debemos tomar en cuenta las condiciones actuales en las que el problema se plantea, no obstante, sin quedar dependiente de esta situación. La pregunta ontológica no debe ser hecha an-históricamente pero tampoco debe cerrarse a los parámetros ontológicos del momento presente. Hay que asumir la herencia histórica, tomarla en cuenta en vista de una apropiación autentica que permita liberar el sentido de esta pregunta con el fin de escapar de las

\footnotetext{
${ }^{1}$ Una primera versión de este articulo ha sido publicado en la revista Alter n²1 en 2013. Sin embargo, éste ha sido revisado y modificado.
} 
aporías a las cuales ella dio lugar a causa de su formulación tradicional, tal como la aporía ontológica (como pensar la unidad del modo de ser de la materia, de la vida y de la mente a pesar de sus diferencias) y la aporía epistemológica (como pensar la correspondencia entre las representaciones de un cogito y la realidad del objeto). Esa es la razón por la cual Hans Jonas se propone a hacer una interpretación de la historia de las interpretaciones del ser en la cual él diagnostica un olvido de la vida. Para exponer esta historia de manera muy breve, Hans Jonas considera que el dualismo del periodo clásico rompe el monismo pan-vitalista de los orígenes y prepara la llegada de dos nuevos monismos, materialista e "idealista." Efectivamente podemos resolver el problema ontológico de la unidad de la res extensa y de la res cogitans gracias a una reducción de la totalidad de la realidad a una de las dos substancias a partir de la cual podemos a continuación derivar la otra.

Podemos hacer tres comentarios a propósito de esta lectura de la historia, sobre la cual echaremos un vistazo. En primer lugar, el dualismo clásico es solamente un resurgimiento, pero conceptualmente más riguroso, de la crisis del período gnóstico ${ }^{2}$ que condujo a romper la unidad cósmica legada por los Griegos. En segundo lugar, este dualismo conserva una actualidad y una cierta influencia, inclusive en el período contemporáneo, ya que él está en el segundo plano de los idealismos y de los materialismos. De hecho, la materia del materialista tiene un sentido solamente gracias a su oposición al espíritu del idealista, y recíprocamente ${ }^{3}$. En tercer lugar, es precisamente a causa de este dualismo de la sombra que estos pensamientos fracasan a resolver el problema del ser. Cada uno toma una substancia como norma ontológica, sin embargo, después debe dar cuenta de la substancia contraria a partir de la primera en nombre de la unidad del ser; lo que da lugar a aporías que no podemos superar: el materialista debe explicar la inmaterialidad del espíritu y el idealista debe justificar la existencia de la realidad extendida y resistente de la materia. Además, cada uno tiene dificultad para explicar el fenómeno de la verdad. El materialista debe mostrar como un trozo de materia puede interiorizarse en una representación que va a copiar la realidad "exterior" y el idealista debe hacernos entender como una representación interior puede dar cuenta de lo que es exterior. Así, debemos rechazar la historia de la ontología que Hans Jonas llama "la ontología de la muerte" a raíz de que ella conduce a un dualismo perjudicial para la comprensión del ser, pero, al mismo tiempo conservar el cuidado de pensar la dualidad, de la cual hacemos la experiencia en nuestra vida. Eso quiere decir que debemos pensar la dualidad pero sin dualismo y, por lo tanto, a partir de un nuevo modelo ontológico.

\footnotetext{
${ }^{2}$ Movimiento religioso de inspiración cristiana y neo-platoniciana desarrollado entre el siglo I y III que afirma que el alma esta prisionera de un mundo malo creado por un dios malo.
}

${ }^{3}$ Ya que sólo un espíritu puro puede concebir una materia meramente extensa. 
Entonces, es solamente al rechazar las categorías metafísicas del alma y del cuerpo, entendidas de manera substancialista, que podremos pensar la vida por ella misma con el fin de desanudar el nudo del ser, es decir, de entender cómo lo que llamamos alma y cuerpo pueden ser unidos y qué sentidos ellos pueden tener. De manera obvia el fenómeno de la vida desmiente el modo de ser de la materialidad, ya que es un lugar de una cierta autonomía o finalidad. Pero él desmiente también el modo de ser del alma, puesto que él es extendido. Este fenómeno hace caduco el dualismo y al contrario la dualidad debe ser comprendida a partir de este fenómeno, que sólo es concreto. Por lo tanto, estamos obligados a concluir que la metafísica con sus categorías debe ser provisoriamente dejada de lado en beneficio de un enfoque fenomenológico, gracias al cual podremos desvelar el modo de ser de la vida. Desde este punto de vista, hace falta destacar un indiscutible aire de familia entre el razonamiento de Merleau-Ponty en La structure du comportement (Merleau-Ponty, 1957) y el de Jonas en The Phenomenon of Life (Jonas, 2001). ${ }^{4}$

Quisiera mostrar, en un primer momento, en qué medida podemos poner énfasis en la convergencia entre estas dos fenomenologías antes de destacar, en un segundo momento, como Hans Jonas podría criticar el enfoque de Merleau-Ponty. ${ }^{5}$ Esta confrontación nos ayudara a definir la especificad del pensamiento de Jonas y, sin duda, la vía de una fenomenología de la vida auténtica.

\section{LA VIDA COMO METABOLISMO Y COMPORTAMIENTO}

Tres observaciones permitirán justificar un tal acercamiento. En primer lugar, ambos autores se encuentran desde el punto de vista de la finalidad del proyecto. Así Merleau-Ponty considera que a la condición de tomar como punto de partida la noción del comportamiento "neutra con respecto a las distinciones clásicas de lo "psíquico" y de lo "fisiológico"" (Merleau-Ponty, 1957, 21), no solamente podremos comprender las relaciones entre la conciencia con la naturaleza sino también podremos redefinir el sentido del alma y del cuerpo. A condición de romper con el dualismo, podremos entender el verdadero sentido de la dualidad y afrontar el cuestionamiento ontológico. La noción de comportamiento viviente se sitúa en un primer plano no solamente porque el organismo viviente es el lugar de una ambigüedad, como lo demuestra el debate entre el mecanismo y el vitalismo, contraria a la explicación física del comportamiento de

\footnotetext{
${ }^{4}$ La primera versión alemana es una traducción, en parte de Hans Jonas, de la versión inglesa y cuenta con algunas modificaciones. Su título es lo de la primera versión inglesa no publicada de The Phenomenon of Life, es decir, Organism and Freedom (publicado en alemán bajo el título: Organismus und Freiheit. Ansätze zu einer philosophischen Biologie). La nueva versión alemana póstuma de 1994 tiene otro título: Das Prinzip Leben, Ansätze zu einer philosophischen Biologie. La versión castellana es una traducción de este último libro (Jonas, 2000).
}

${ }^{5}$ Me parece que nunca Hans Jonas hace referencia a Merleau-Ponty. 
la materia o a la explicación psicóloga de la conciencia, que unas veces es vista como una cosa material y otras como una cosa espiritual; sino también porque el ser humano es el principio de la investigación filosófica, y no podemos entender sus posibilidades de comprender ni de actuar sin hacer referencia a su actividad nerviosa, gracias a la cual éste se relaciona con su entorno. Esta noción de comportamiento viene del behaviorism y tiene el mérito de desespiritualizar al humano. No obstante, como lo nota Merleau-Ponty, esta despsicologización no debe conducir a un razonamiento inverso de remecanización o de rematerialización. El comportamiento debe quedar neutro en relación con esta división ontológica de la materia y del espíritu para que entendamos el hombre como "debate y "explicación” perpetua con un mundo físico y con un mundo social." (Merleau-Ponty, 1957, p. 21).

Hans Jonas también propone una interpretación existencialista de los hechos biológicos que escapa de la oposición del objeto y del sujeto. Según él, el espíritu no puede ser entendido sin una base orgánica y el cuerpo no puede ser comprendido como un sencillo mecanismo. Debemos tomar en cuenta el testimonio del hombre respecto del cuerpo viviente, ya que este comportamiento recapitula, por decirlo así, las diferentes regiones ontológicas de la materia, de lo orgánico y del espíritu. Como dice Hans Jonas (2000), pp. 39-40:

Si se da a la cuestión su auténtico sentido, quizá sea verdad que el hombre es la medida de todas las cosas: no en virtud de la legislación promulgada por su razón, ciertamente, pero sí en virtud del paradigma de su totalidad psicofísica, la cual representa el máximo de completitud y concreción ontológica que conocemos. Desde ese paradigma, los tipos de ser van siendo determinados reductivamente mediante progresivas sustracciones ontológicas, hasta llegar al mínimo de la mera materia elemental en lugar de que la materia más completa vaya siendo constituida desde esta base mediante añadidos acumulativos). ${ }^{6}$

Además es porque el hombre pertenece al reino de la vida que él es capaz de reconocer los otros seres vivientes en su entorno. Como Hans Jonas (2000), p. 128, explica:

El observador de la vida tiene que estar preparado por la vida. (...) Solo gracias a la interpolación de la identidad interior así permitida es posible concebir el mero factum morfológico de la continuidad metabolizante como acto incesante, es decir, concebir el hecho de estar prolongado como autoprolongación.

\footnotetext{
${ }^{6}$ En este caso podemos hablar de un antropomorfismo positivo, ya que el punto de vista humano no es un obstáculo para conocer la vida. Como el ser humano hace parte de la vida, él tiene informaciones sobre ella. Al contrario, la ciencia denunció el punto de vista humano como siendo antropomórfico cuando se trataba de conocer una realidad en sí misma. Por ejemplo, Descartes condenaba la visión antropomórfica del animal. No debemos proyectar sobre él nuestra propia experiencia humana de la finalidad, del proyecto, de la voluntad. Y por eso debemos interpretar el animal mecánicamente. Pero la crítica al antropomorfismo presupone una división de la realidad entre un sujeto y un objeto; el fenómeno de la vida que no es sujeto ni objeto, desmiente esta división.
} 
Ahora indiquemos el segundo punto de convergencia entre los dos autores. Más allá de la finalidad del proyecto, es decir, además de la voluntad de poner la pregunta de la vida al centro de la reflexión ontológica, Hans Jonas y Merleau-Ponty se juntan en la descripción del fenómeno de la vida. Efectivamente, independientemente del mecanismo de la ciencia y de la reflexión de la conciencia, Merleau-Ponty comprende el comportamiento a partir de las nociones de estructura y de forma. La forma se aplica al campo físico, biológico y psicólogo, pero según tres estructuras diferentes. En lugar de oponer la materia y la cantidad, la vida y su orden, el espíritu y su valor, Merleau-Ponty explica que es una misma forma cuya estructura varía al poner en primer plano tal o cual carácter (cantidad, orden, valor) según el orden fenomenal considerado. La diferencia estructural debe ser entendida en términos de grados de integración de la forma, de manera que la individualidad se realice cada vez más según qué tomamos en cuenta la estructura física, biológica o psicóloga. Esta descripción tiene una doble ventaja. Ella permite rechazar el reduccionismo, por ejemplo, el reduccionismo materialista, a raíz de que no podremos decir que la forma orgánica o psicóloga es de la misma naturaleza que la forma física. Pero ella permite dejar de lado también el separatismo ontológico que agrieta la naturaleza, al afirmar la diferencia radical de estas estructuras entre ellas. La estructura biológica ocupa un espacio privilegiado en el enfoque de Merleau-Ponty, puesto que ella permite echar una luz sobre la dimensión dialéctica ${ }^{7}$ del ser vivo. Mientras la forma física resulta de un equilibrio con las condiciones exteriores, y la reacción del sistema material tiene la ambición de reducir una tensión, de caminar hacia un estado de reposo, la forma viviente, por su parte, no obtiene su equilibrio en relación con las condiciones actuales sino con las condiciones posibles que el sistema tiene precisamente por vocación a llevar a la existencia. El organismo no se contenta con adaptarse de manera mecánica a las condiciones actuales, pero él se ajusta a ellas al preferir ciertos comportamientos a otros. Con la vida, el ser se abre a la expresión de una significación que traduce la orientación de una posibilidad orientada hacia la exterioridad que él contribuye a esclarecer. Como dice Merleau-Ponty (1957), p. 228:

El fenómeno de la vida aparecía pues en el momento en que un fragmento de extensión, por la disposición de sus movimientos y por la alusión que cada uno de ellos hace a todos los otros, se replegaba sobre sí mismo, se ponía a expresar alguna cosa y a manifestar hacia afuera un ser interior.

Ahora podemos insistir en la comunidad de vista con Hans Jonas. Con Hans Jonas también debemos rechazar el enfoque substancialista si queremos entender las diferencias ontológicas regionales en el seno de un enfoque unitario del ser. Materia, vida y espíritu no son unidades ontológicas autónomas que desmiembren la

\footnotetext{
${ }^{7}$ La vida niega en parte "la realidad" para afirmarse al imponer sus propias posibilidades.
} 
unidad de la naturaleza sino ordenes de significación en el seno de una sola y misma concepción del ser cuyas diferencias demuestran un progreso cada vez mayor de la individualidad hacia a un mundo cada vez más abierto. Desde el animal hacia el hombre es siempre una misma vida que se afirma, salvo que el sí mismo se afirma cada vez más y que los horizontes se amplían. Así, gracias al poder de la motricidad, de la percepción y de la emoción, el animal puede abrir horizontes espaciales y temporales, lo que faltaba a la planta. Así, gracias a la imaginación y al poder simbólico, el hombre puede acrecentar su libertad y reflexionar sobre sí mismo. Por el medio de la imaginación el hombre va a desarrollar el poder técnico, pero también el conocimiento de sí mismo. Este progreso de la vida, manifiesta la trascendencia de un sí mismo abierto al mundo, al surgimiento de posibilidades, sobre el cual Merleau-Ponty ya había sabido echar luz. Aquí también el término de dialéctica conviene, puesto que la libertad del ser vivo se afirma en un debate cada vez más peligroso con el entorno a medida que la vida progresa. Efectivamente, el animal se abre al mundo, se proyecta hacia él, pero también se inscribe en él mientras que el hombre es capaz de transformarlo. Quizás esta dimensión dialéctica de la vida es aún más fuerte en la filosofía de Hans Jonas que en la de Merleau-Ponty, ya que la exigencia de libertad es, según su juicio una necesidad. Si el animal o el hombre no ejercen su libertad, es su vida como animal o como hombre la que está en juego. Podemos añadir que la vida toma cada vez más riesgos. Mientras la planta encuentra todo lo que ella necesita en el mismo lugar, el animal está condenado a buscar lo que falta con el riesgo de no encontrarlo. En cuanto al hombre, su poder imaginativo es una suerte para su libertad, pero él corre el riesgo de perderse en la ficción. De cualquier manera, en la filosofía de Hans Jonas también, la noción de forma permite unificar los diferentes órdenes de fenómeno. Hay una forma para el ser físico como para el ser vivo, pero debemos distinguir a los dos. La forma del primero resulta de sus componentes materiales mientras la forma del segundo da cuenta de la renovación de estos componentes. La soberanía o la independencia de esta forma respecto a la materia exige la sustitución de las entidades materiales. Esta sustitución es la vida misma, puesto que su conservación estática significaría la muerte del organismo, su vuelta a la materialidad. Entonces para renovarse, para hallar una materia que le permite mantenerse, la forma debe ser el principio de apertura al mundo extranjero y a sí misma, con el fin de distinguir en el entorno lo que le convenga y lo que debe rechazar. Desde este punto de vista, el metabolismo es el verdadero equivalente jonasiano del rol que juega la noción de comportamiento en la filosofía de Merleau-Ponty y permite entender el sentido de la vida. El ser vivo debe cambiarse gracias a intercambios con el entorno para ser el mismo. En Jonas como en Merleau-Ponty, la trascendencia de la vida permite abrir un mundo, hacer surgir horizontes que permiten a una individualidad afirmarse. Para los dos, la vida 
no es un enigma sino lo que nos permite esclarecer el enigma del ser, constituido de diferencias que no comprometen su unidad.

Evoquemos el tercer punto de convergencia en cuanto al método de los dos autores. Para ambos filósofos el punto de vista del sujeto encarnado permite interpretar el fenómeno de la vida como vida. Jonas reivindica el valor metodológico del antropomorfismo. Ya que él rechaza la división ontológica del sujeto y del objeto, ya no podemos denunciar el punto de vista del hombre sobre la vida con el pretexto que ello sea una proyección subjetiva sobre una realidad objetiva. La forma viva desmiente esta división entre sujeto y objeto. Puesto que el hombre se sabe cómo ser vivo, él puede reconocer alrededor de él otros seres vivos. Este reconocimiento pasa por una interpolación del acto de renovación de sí mismo, característico de la actividad metabólica, sobre el otro ser vivo. Esta interpolación no es la proyección de la finalidad, característica del espíritu, sobre otro cuerpo, sino la identificación al exterior de sí mismo, en otro organismo, del mismo tipo de actividad metabólica que descubrimos en nosotros mismos. Esa es la razón por la cual Jonas concede una importancia esencial al testimonio corporal, como ya hemos visto. Mi cuerpo es la articulación del intensivo y del extensivo, la unidad de la interioridad y de la exterioridad. Cito a Jonas (2000), p. 33:

Pero precisamente el cuerpo vivo, el organismo, presenta esa autotrascendencia en ambos sentidos (...). Hemos de describirlo como extenso e inerte, y a la vez como capaz de sentir y de querer, pero ninguna de las descripciones puede llegar a término sin traspasar el límite que la separa de la otra y sin prejuzgarla.

El alma existe solamente en relación con el cuerpo y el cuerpo vivo existe solamente en relación con el alma. El sentido de mi cuerpo es el punto de partida de una comprensión de la vida en general y de su apertura al mundo.

A primera vista, el enfoque es el mismo en la filosofía de Merleau-Ponty. Los dos primeros capítulos de La structure du comportement tienen como finalidad la de proceder a una crítica interna del enfoque behaviorista del ser vivo, al mostrar que él no puede dar cuenta ni de los comportamientos inferiores ni de los comportamientos superiores puesto que él reanuda con el mecanicismo científico. Esta denuncia del mecanicismo científico habría sido operado por la vía corta de la reflexión ${ }^{8}$ pero esta vía larga, la que opera una mera critica detallada del enfoque behaviorista del ser vivo, permitía rechazar el mecanicismo al ratificar la insuficiencia del punto de vista reflexivo: el comportamiento vivo, que no es mecánico, tampoco no pertenece al orden de la idea, de ahí la importancia de la noción de forma que permite neutralizar la oposición entre la materia y la idea y, al mismo tiempo, de pensar las

\footnotetext{
${ }^{8}$ Eso quiere decir que habríamos podido rechazar el mecanismo al confiarnos a la experiencia cartesiana de la reflexión que descubre que no somos solamente una máquina sino también un espíritu.
} 
diferencias estructurales entre la forma física, viviente y psíquica. Así, este enfoque indirecto del comportamiento vivo de los primeros capítulos prepara el enfoque más directo del tercer capítulo, en el cual la forma viva es descrita por ella misma y por comparación con la forma física y la forma psicológica.

Sin embargo, a pesar de que la crítica interna de la ciencia sea privilegiada y después la descripción de las diferentes estructuras de la forma, debemos reconocer que esta descripción sólo es posible a condición de que el sujeto tome en cuenta su propia encarnación, esto porque el sujeto entiende a partir de su cuerpo que el reconocimiento de las otras formas vivas es posible y que el organismo percibido es comprendido como percepción de un organismo vivo. La percepción del organismo no es una apariencia sino un fenómeno que tiene su propia significación, y ello no gracias a un razonamiento por analogía, sino gracias a una interpretación inmediata, puesto que el otro vivo es directamente entendido como vivo y no gracias a una comparación. Entonces Merleau-Ponty como Jonas entienden la vida de los seres vivos a partir del punto de vista del sujeto percibiendo, pero con la condición de entenderlo después de una reducción de naturaleza fenomenológica que es una inversión del movimiento natural de la conciencia. En lugar de entender el sujeto como parte de un mundo objetivo, debemos entenderlo como abierto al mundo según una estructura intencional. Eso aparece con fuerza en el último capítulo de La structure du comportement. De hecho, la crítica interna de las ciencias de los capítulos anteriores supone esta experiencia de la vida, entendida como percepción "vivida" del fenómeno de la vida. Así, el orden humano de la conciencia no es un orden que se añade al orden físico y biólogo, sino que él es la condición de posibilidad de estos órdenes. Esta conciencia es una conciencia encarnada, es decir perceptiva, y no puramente intelectual. Para ella, el cuerpo no es una cosa ni una idea sino el medio para abrirse al mundo9. Gracias a él puedo tener acceso a la profundidad del mundo y escapar de la alternativa entre el subjetivismo relativo y el objetivismo ingenuo. La cosa percibida no es en sí ni una construcción del espíritu. Ella se da a través de los perfiles (Abschattungen) que la manifiestan. La parcialidad de ellas no significa que nuestra percepción sea relativa o falsa, sino que la cosa es

\footnotetext{
${ }^{9}$ El Profesor Luis Flores Hernández, quien conduce una reflexión propia, nutrida por su conocimiento de la fenomenología y en particular la de Husserl, muestra en "Fenomenología de la corporalidad y de la intercorporalidad" (Flores, 2010, pp. 169-175) que debemos interpretar el tejido intencional del cuerpo vivido a partir de cuatro direcciones, las funciones cognitiva, deóntica, sintomática y estética, lo que permite describir el mundo de manera realmente vivido. Además, en "Fenomenología de la espacialidad en el horizonte de la corporalidad" (Flores, 2003, pp. 265-269), él destaca que "el espacio sagrado es una modalidad de espacio vivido, en el cual las diferentes intencionalidades se articulan en torno a lo sagrado, lo numinoso, o más específicamente, a Dios." Por lo tanto, podríamos añadir una quinta dimensión al mundo espacialmente vivido que es también una dimensión privilegiada puesto que ella unifica de manera particularmente íntima las otras dimensiones. Sin embargo, como lo señala el autor, la dimensión cognitiva no tiene tanta importancia en cuanto a la descripción del espacio sagrado vivido.
} 
trascendente. El perspectivismo es una ley de la cosa misma. Entonces no hay tres cosas perteneciendo a tres regiones diferentes de la realidad - el alma, el cuerpo y la cosa - sino un campo único: las intenciones del alma son expresadas por el cuerpo que nos abre al mundo en la percepción. Es porque yo sé que soy abierto al mundo gracias a mi cuerpo que reconozco inmediatamente en este mundo otros cuerpos que tienen esta apertura al mundo.

Hemos visto que había convergencia entre el proyecto de Jonas y de Merleau-Ponty desde el punto de vista de la finalidad del proyecto, de su contenido y de su método. Sin embargo, quisiéramos mostrar ahora en qué medida sería posible criticar el enfoque de Merleau-Ponty y en qué medida la solución de Jonas podría ser vista como un progreso desde el punto de vista de una fenomenología de la vida.

\section{HaCia unA FENOMENOLOGÍA DE LA VIDA}

De hecho, podríamos decir que la filosofía de Merleau-Ponty queda dependiente del dualismo del sujeto y del objeto, lo que reconduce el método del idealismo, en desmedro de su pretensión a pensar la unidad del ser. Es lo que podemos constatar en el último capítulo de La structure du comportement, en el cual Merleau-Ponty mantiene un punto de vista trascendental, al referir la aprensión del comportamiento al descubrimiento de una significación por la conciencia (MerleauPonty, 1957, 256-257). Es cierto que esta conciencia es perceptiva y no intelectual, cortada de sus raíces vitales. Es cierto que la división del alma y del cuerpo no es "real" sino resuelta de una distinción entre dos órdenes de significación. La percepción "vivida" no puede ser interpretada en términos de representación psíquica ni de explicación causal. No obstante, todo pasa como si la reducción recondujera el presupuesto de una conciencia que podía existir aparte del cuerpo y como si ella impusiera su modo de ser a otros seres descritos. En lugar de ser descrita por ella misma y de dar lugar a continuación a la conciencia, en lugar de describir la génesis de la conciencia a partir de la vida, la conciencia viene en primer lugar e impone su modo de ser a la vida. Es lo que confirma el punto de vista desarrollado en $L a$ Phénoménologie de la perception que trata de entender nuestra apertura hacia el mundo a partir de la experiencia del cuerpo vivido. Es cierto que al desobjetivar el cuerpo - lo que es la meta de la primera parte del libro - se da el medio de entender el mundo percibido y el para-sí (respectivamente en las segunda y tercera partes) independientemente de la separación entre el punto de vista de la explicación y el de la reflexión. No obstante, al no describir el cuerpo como idea ni como cosa, MerleauPonty queda dependiente de ambos conceptos, lo que él reconocerá ulteriormente (Merleau-Ponty, 1964, p. 253). Por lo tanto, en lugar de describir el surgimiento de la conciencia a partir de la vida, la descripción de la vida está hecha a partir del 
punto de vista de la conciencia perceptiva entendida desde la oposición del alma y del cuerpo.

Por eso, el pensamiento de Hans Jonas es un progreso en cuanto a la definición de una fenomenología de la vida. Al inspirarse de la pareja conceptual de Whitehead entre la "inmediatez presentativa" y la eficiencia causal, ${ }^{10}$ Jonas consigue justificar a partir de la vida la tendencia inherente a la percepción a objetivar el ser y a entenderse como acto de un sujeto contemplativo. Jonas comparte con Merleau-Ponty la voluntad de traspasar la antinomia de la explicación objetiva y de la definición intelectualista de la percepción, pero él se da los medios de superar este dualismo de otra manera que la del recurso a una operación de reducción que queda dependiente del punto de vista de la conciencia, puesto que él describe a la raíz, quiere decir en su génesis, el principio según el cual la vida es condenada a ilusionarse sobre ella misma. En otros términos, es porque la vida se abre al mundo a través de la percepción y es porque la percepción es originariamente consagrada a la objetivación que el ser vivo percibiendo se representa como sujeto consciente. Es del fondo de la vida misma que la dualidad surge y que su verdadero sentido se disfraza detrás de la figura del dualismo. El ensayo VI de The Phenomenon of Life no se dedica a la fenomenología de la percepción sino a la fenomenología de los sentidos y, gracias a una comparación de los sentidos entre ellos, este capítulo tiene por función la de mostrar que la visión se distingue de los otros sentidos a causa de su poder de distanciación y de neutralización causal. Mientras la percepción en general permite al ser vivo abrir un horizonte espacial, debemos reconocer que la primacía de la visión tiene tendencia a borrar el mundo en beneficio de una visión objetiva de la naturaleza, tal como un sujeto desatado del mundo puede representárselo, a causa de su falta de implicación en él. Efectivamente, la visión borra el debate entre la cosa y el sujeto, entre el sujeto que se implica en el mundo y la cosa que hace pregunta al sujeto. Esta idealización del mundo puede ser una ventaja desde el punto de vista de la explicación científica, ya que ella nos da una visión sin profundidad, sin oscuridad. Sin embargo, es un defecto desde el punto de vista ontológico. Por lo tanto, hay que criticar la dimensión idealista de la percepción visual para reanudar con el sentido originario de la percepción que deriva de la apertura del mundo de la vida. No se trata de renunciar a ver, sino más bien de no reducir la percepción a la visión y de integrar la visión en el marco de una percepción más amplia para destituir su monopolio. Bajo esta condición, la experiencia táctil esta revalorizada, puesto que ella es la ocasión de aproximarse del movimiento de la vida, por ejemplo en la experiencia del choque, en el cual una fuerza se da a sentir y gracias a la cual

\footnotetext{
${ }^{10}$ Que no puedo desarrollar aquí. Whitehead explica que la visión idealiza nuestro conocimiento de la realidad (es la "inmediatez presentativa") mientras que la experiencia más directamente corporal nos da una idea de esta realidad (Whitehead, 1978, Part II, chapter VIII) y Jonas (2000), p. 47.
} 
se desvela de manera correlativa mi presencia y la del mundo, mi compromiso hacia el mundo y "el efecto" del mundo sobre mí. Por lo tanto, mientras la visión es par excellence el sentido que hace posible nuestra relación objetiva al mundo, el tacto permite experimentar las fuerzas del mundo de manera privilegiada. Pero es claro que esta experiencia de la fuerza no debe ser interpretada en términos mecanicistas, ya que ella crea al contrario las condiciones gracias a las cuales una objetividad podrá ser construida. De hecho, la experiencia del mundo que hago en el tacto, que supone el movimiento corporal, es la condición originaria a partir de la cual la visión podrá constituir la objetividad de la naturaleza. Es porque mi cuerpo tocando se mueve que puedo sintetizar, por una parte, los datos del objeto proporcionados por un solo y mismo sentido y, por otro lado, los datos de los diferentes sentidos. A pesar de que la visión debe ser considerada como el sentido más objetivante, Jonas estima que ella no podría jugar su papel sin la experiencia de un sí mismo corporal moviéndose en un espacio que él abre, por este medio, y gracias al cual podemos identificar objetos. Jamás, por ejemplo, tendríamos el sentimiento de la perspectiva, necesaria para percibir objetos, si no hubiéramos tenido anteriormente la experiencia del movimiento.

Por lo tanto, podemos atribuir la tendencia idealista al predominio de la visión que oculta la raíz vital de nuestra apertura al mundo y habría que mostrar como este monopolio tiene un efecto en la propia fenomenología de la percepción de Merleau-Ponty después de La Structure du comportement, como lo muestra por ejemplo Françoise Dastur cuando explica que Merleau-Ponty restablece la simetría entre la visión y el tacto ${ }^{11}$ que Husserl había abolido en beneficio del tacto (Husserl, 1952, §36-37). Sin embargo, si esta interpretación es exacta, es necesario identificar aún más profundamente la causa del olvido de la vida y su recubrimiento por una aprensión substancialista debida al modelo óptico de nuestro pensamiento, como si la vida debía ser un objeto estudiado por el científico o un vivido de la conciencia fenomenológica sin dimensión encarnada. Efectivamente es cierto que la concepción óptica del ser y de la vida conduce a olvidar la dimensión existencial de la vida. Pero debemos justificar por qué seguimos este movimiento de la vida hacia una lectura óptica de la vida. Debemos dar la razón por la cual obedecemos al dominio de la visión en lugar de reintegrar sus informaciones en el campo más amplio de la percepción general. En otros términos, ¿por qué nos gusta tanto confiarnos a la visión más que al tacto y al sentimiento de movimiento? ¿Porque preferimos la acción del tipo técnico, que hace posible la visión a distancia, y la "contemplación”,

\footnotetext{
${ }^{11}$ Como Françoise Dastur dice: "Merleau-Ponty [restaura] el paralelismo entre el ver y el tocar que cuestionó Husserl" ("Merleau-Ponty [restaure] le parallélisme entre le voir et le toucher que contestait Husserl") (Dastur, 1988, p. 137). Ella habla de "un privilegio exorbitante" («un privilège exorbitant» de la visión) en el marco de la filosofía de Merleau-Ponty.
} 
que hace posible la desaparición de las fuerzas causales en la percepción visual, y no el movimiento de la existencia? ¿Porque manipular técnicamente o teóricamente más que existir? Quisiéramos sugerir que el olvido de la existencia vital se debe a lo que ya llamamos con Jonas "la ontología de la muerte." Eso quiere decir que hay un movimiento de la historia de la filosofía dirigido por una tendencia dualista. Pero esta separación del cuerpo y del alma, de lo material y de lo espiritual tiene un origen en una concepción exterior de la muerte. Ella es vista como el fin de la vida, como el contrario de la vida, lo que la niega. Ya que la muerte es el fin del cuerpo orgánico, solo el alma puede ser concebida como la causa de la sobrevivencia. La huida del alma explica porque - de repente - el cuerpo deja de vivir. Sin esta hipótesis la muerte sería incomprensible dentro del sistema panvitalista de los orígenes que afirma que todo está vivo. Esta separación contribuye fuertemente a entender el dualismo del período clásico. Es porque concebimos la muerte como una interrupción inexplicable de la vida y exterior a ella que somos conducidos a pensar en términos dualistas según Jonas.

Por lo tanto, debemos reintegrar la muerte dentro de la vida y pensarla no solamente como una negación de la vida sino también como una condición de la vida. La muerte tiene una dimensión dialéctica, y lo que niega la vida es también lo que le permite afirmarse. Cito a Jonas (1998), p. 19:

La vida no es mortal a pesar de ser vida, sino por el hecho de serlo, por su constitución originaria; porque la relación entre forma y materia en que la vida se basa, tiene este carácter revocable y carente de garantías.

El ser vivo se cuida de sí mismo a causa del riesgo de la muerte. Y este riesgo es la condición de su apertura al mundo. Su precariedad es su suerte de existir. Se trata de la "gran muerte que cada uno tiene en sí", de "la fruta que está al centro de todo" (Rilke, 1972), como decía Rainer Maria Rilke, y que debemos oponer a la pequeña muerte que es exterior y accidental. Cuando Jonas nos invita a reintegrar la dimensión visual de nuestra existencia en el campo de una experiencia perceptiva más amplia, es decir, cuando pone el énfasis sobre el tacto y el movimiento, me parece que es una invitación a experimentar nuestra relación al mundo en términos de fuerza, sea del impacto del mundo sobre el ser viviente o bien sea el del ser viviente sobre el mundo. Además esta experiencia es la experiencia de nuestra precariedad y de la fragilidad del ser vivo que puede morir y que, por esta razón, abre horizontes, abre un mundo. La experiencia de la contingencia de nuestra vida nos abre a la experiencia de la contingencia de la vida. Al contrario, es porque dejamos de lado esta precariedad de la existencia, es porque somos determinados por una concepción exterior de la muerte, que reducimos la percepción a la visión; que entendemos la percepción como un tipo de visión. El descubrimiento que la muerte hace parte de la vida es al mismo tiempo una conciencia 
de la parcialidad de la visión. Nuestra encarnación supone la precariedad en sí de nuestra existencia. Al contrario, la visión adquiere un carácter absoluto a causa de este olvido. Esa es la razón por la cual Hans Jonas puede decir que la visión prepara la contemplación y la idea de eternidad.

Podríamos objetar que Merleau-Ponty no ignora esta importancia de la muerte para dar cuenta del fenómeno de la vida. Al fin de La structure du comportement, él muestra que debemos oponer una estructura efectiva y la significación ideal a propósito de la significación verdadera de nuestra vida. Así debemos distinguir una liberación real y una liberación ideal. La segunda nos invita al quietismo y a una existencia separada, mientras la primera propone adquirir la conciencia de sí mismo bajo la condición de una reapropiación de los conocimientos sociológicos, psicológicos etc. que determinan nuestro pensamiento de la vida. La conciencia no es inmediata, sino que supone de tomar en cuenta nuestra encarnación y todo lo que nos determina. No significa que la vida humana es mecánicamente determinada. Solo quiere decir que es necesario rechazar la alternativa reflexión/mecanismo, al entender que la conciencia no es pura, inmediata y exige de tomar en cuenta las condiciones que la determinan sin necesitarla. En este contexto, Merleau-Ponty concede importancia a la muerte. Efectivamente, ella permite disolver las significaciones eternales que una conciencia pura podría tener en beneficio de la conciencia de la contingencia de los vividos (Merleau-Ponty, 1957, p. 240). La muerte no es el reino del absurdo, sino al contrario la condición de una vida auténticamente vivida, ya que nuestra vida no debe encerrarse en una significación para siempre, pero exige de ser puesto en cuestión. No implica necesariamente que debamos afirmar que la experiencia de la eternidad no existe, sino que ésta debe integrar la conciencia de la muerte. Además, la integración de la muerte en la consideración de la vida permite de distinguir entre el amor a la vida y el apego a la existencia orgánica. Merleau-Ponty quiere probablemente decir que podemos incluir la posibilidad de sacrificar nuestra vida orgánica para salvar nuestra vida, más bien el sentido de nuestra vida, si las circunstancias lo exigen. El amor a la vida puede exigir el poner fin a nuestra vida orgánica, o mejor dicho poner en peligro nuestra existencia orgánica. Entonces, la muerte no significa la absurdidad de nuestra vida, sino que tomarla en cuenta es la condición de la significación autentica de esta vida. Perder la vida es una posibilidad que es una dimensión continua de esta vida. Desde este punto de vista, podríamos decir que Merleau-Ponty no ignora la exigencia jonasiana de integrar la muerte en nuestro equipamiento ontológico para comprender la esencia de la vida en su dinámica.

Sin embargo, estamos obligados a reconocer que esta comprensión de la muerte queda dependiente de un cierto dualismo. En otros términos, a pesar de que Merleau-Ponty parece integrar la muerte en la vida, él reconduce la ontología de la muerte que separa el alma y el cuerpo. En efecto, primero la vida de que él habla 
queda la vida de la conciencia. El pensamiento de la muerte le permite romper con una visión eternal de la significación en beneficio de la conciencia de la contingencia de sus contenidos. Pero él no evoca el origen de la muerte y la razón por la cual ella tiene este efecto sobre nuestra conciencia. El no muestra que pensamos en la muerte a causa de la mortalidad de nuestro cuerpo. El no muestra que el pensamiento de la contingencia viene de la precariedad de nuestra vida que es también orgánica. Al contrario, él abre la posibilidad de una vida eterna que continua a pesar de nuestra mortalidad, como si la conciencia tuviera la posibilidad salvarse de su finitud que Jonas considera, desde el punto de vista ontológico, como definitivo. No obstante entender que la muerte pertenece a la vida es un medio esencial para entender que venimos de la tierra y que vamos a la tierra. Es un recuerdo al estatuto material de nuestra vida ${ }^{12}$. Es un factor de desidealización. Sería entonces a causa de este olvido que podríamos explicar el resto de idealismo que hemos diagnosticado en la filosofía de Merleau-Ponty y su tendencia a recargar el papel de la visión.

Quisiéramos concluir al decir que, al contrario, Jonas integra plenamente la muerte dentro de la vida como su negación y también como su condición. Es porque la vida puede morir que ella debe abrirse, gracias a horizontes y sin que podamos distinguir una substancia psíquica o una substancia corporal. En efecto, esta apertura toma la forma de un movimiento - lo que llamamos la vida - que es capaz de percepción. Se trata de una fuerza que puede percibir, abrir horizontes espaciales y temporales. Por lo tanto, nos parece importante para el desarrollo de una fenomenología de la vida romper con el dualismo del alma y del cuerpo en beneficio de un pensamiento unitario que no sea substancial. Y un elemento esencial para seguir esta vía es la de comprender la vida a partir de la posibilidad de su muerte, de una muerte que no es exterior a la vida sino su condición esencial.

\section{BILBIOGRAFÍA}

Dastur, Françoise (1988). "Monde, chair, vision ». En : Maurice Merleau-Ponty. Le psychique et le corporel. Colette, Jacques y Tymieniecka, Anna-Teresa (eds.). Paris : Aubier.

Flores Hernández, Luis (2010). "Fenomenología de la corporalidad y de la intercorporalidad". En: Fenomenología y Hermenéutica. Actas del II Congreso Internacional de Fenomenología y Hermenéutica. Eyzaguirre Tafra Sylvia y Johnson Muñoz, Felipe (eds.). Santiago: Universidad Andrés Bello, pp.169-175

\footnotetext{
${ }^{12}$ Este enfoque ontológico debe ser complementado por un enfoque metafísico que no desarrollamos aquí. En efecto, Hans Jonas elabora una teología a partir de tal concepción de la vida (Jonas, 20001, capitulo 12).
} 
Flores Hernández, Luis (2003). "Fenomenología de la espacialidad en el horizonte de la corporalidad”. Teología y Vida, Vol. XLIV, pp. 265-269.

Husserl, Edmund (1952). Ideen zu einer reinen Phänomenologie und phänomenologischen Philosophie, Zweites Buch Phänomenologische Untersuchungen zur Konstitution. Biemel, Marly (ed.) The Hague, Netherlands: Martinus Nijhoff.

Jonas, Hans (2001). The Phenomenon of Life. Evanston, Illinois: Northewestern University Press.

Jonas, Hans (2000). El principio de vida: hacia una biología filosófica. Mardomingo, José (trad.). Madrid: Editorial Trotta (Original en alemán : Jonas, Hans (1973). Organismus und Freiheit. Ansätze zu einer philosophischen Biologie. Frankfurt am Main: Suhrkamp, y luego: Jonas, Hans (1994). Das Prinzip Leben, Ansätze zu einer philosophischen Biologie. Franckfurt am Main: Suhrkamp).

Jonas, Hans (1998). Pensar sobre Dios y otros ensayos. Ackermann, Angela (trad.). Barcelona: Herder. (Original en alemán: Jonas, Hans (1994). Philosophische Untersuchungen und metaphysische Vermutungen. Frankfurt am Main: Suhrkamp).

Merleau-Ponty, Maurice (1957). La Estructura del comportamiento. Alonso, Enrique (trad.). Buenos Aires: Librería Hachette. (Original en francés : Merleau-Ponty (2002). La structure du comportement. Paris: P.U.F.).

Merleau-Ponty, Maurice (1964). Le visible et l'invisible. Paris: Gallimard.

Rilke, Rainer Maria (1972). "Das Buch von der Armut und vom Tode”. En: Das Stundenbuch, Frankfurt: Insel Verlag.

Whitehead, Alfred North (1978). Process and Reality. New York: The Free Press. 